\title{
Exploring the Theory and Teaching Practice of Integrating Ideological and Political Elements Into the Classroom
}

--Take Linear Algebra as an Example

\author{
XuJuan $^{1, \mathrm{a}}$, WangYing ${ }^{1, \mathrm{~b}^{*}}$ \\ ${ }^{1}$ School of Mathematics and Statistics, Linyi University Linyi China \\ axujuan0120@126.com \\ b*lywy1981@163.com
}

\begin{abstract}
The curriculum carries ideological and political education, which is embodied in the curriculum. We aim to fully tap the ideological and political elements contained in the course of linear algebra, and form the first classroom with teaching as the main position and the online second classroom with "reading book club" as the main form in practice, which complement each other and complement each other. Taking a specific classroom teaching as an example, this paper discusses how to integrate knowledge teaching, ability training and value shaping, so as to achieve the good effect of moistening things and educating students.
\end{abstract}

Keywords: Course Ideological and political education, linear algebra, first class

\section{探索思政元素融入课堂的理论与教学实践 \\ - 以《线性代数》课程为例}

\author{
许娟 1 , a 王颖 $1, \mathrm{~b}^{*}$ \\ 1 临沂大学数学与统计学院, 临沂, 山东, 中国 \\ axujuan0120@126.com \\ b*lywy1981@163.com
}

\section{摘要}

课程承载思政, 思政寓于课程。我们旨在充分挖掘《线性代数》课程中蕴含的思政元素, 实践中形成以教学为主 阵地的第一课堂和以 “阅读书会” 为主要形式的线上第二课堂, 两者相辅相成、相得益彰。并以一节具体的课堂教 学为例探讨如何将知识传授、能力培养和价值塑造三者不动声色地融为一体, 以期达到春雨润物, 育人于心的良 好效果。

关键词: 课程思政, 线性代数, 第一课堂

\section{1. 前言}

2020 年 5 月教育部印发了《高等学校课程思政 建设指导纲要》的通知, 通知中指出, 要紧紧抓住教 师队伍这个主力军、把课程建设当作主战场、课堂教 学为主渠道, 让所有的高校、所有的教师、所有的课
程都能承担好育人责任, 要求守好一段渠, 种好责任 田，使各类课程与思政课程同向同行，将显性教育和 隐性教育相统一, 形成协同效应, 构建全员全程全方 位育人大格局。 
作为一线教师的我们应该把自己所教课程的思 政育人功能作为一种日常教育理念, 摈弃各种顾虑杂 念, 积极识变、应变、求变、乐变, 让课程融入思政的 理念入脑、入心、入实践。

\section{2. 构建 “第一、第二课堂”, 找准切入点, 增加思政性，提升创新性}

以习近平同志为核心的党中央高度重视爱国主 义教育, 固本培元、凝心铸魂, 而且要把青少年作为 爱国主义教育的重中之重, 将爱国主义精神贯穿于学 校教育全过程，推动爱国主义教育进课堂、进教材、 进头脑, 要紧紧抓住青少年阶段的年龄特点, 充分发 挥课堂教学的主渠道作用, 发挥学生在课堂教学中主 体作用, 依据不同的教学内容, 采取互动式、启发式、 探究式教学等多种教学方式, 增强教学内容的思想性、 理论性和针对性, 加强爱国主义教育, 在教育教学过 程中, 潜移默化, 润物细无声, 引导学生树立国家意 识、培养增进爱国情怀, 虚功实做, 久久为功。

\section{1. 立足课程内容, 深入挖掘思政元素}

《基础学科拔尖学生培养计划 2.0 》对我们做了 战略警醒, 落后就要挨打, 领跑才能复兴, 并对基础 学科进行再认识: 基础学科是科技创新的母机、战略 安全的底牌、国家富强的血脉。

爱国主义是中华儿女最自然、最朴素的情感, “对 每一个中国人来说, 爱国是本分, 也是职责, 是心之 所系、情之所归。”[1-3] 授课过程中, 教师要引领学生 主动把自己的理想与祖国需要联系在一起, 弘扬正确 的价值理念和道德观念。

通过阅读与课程相关的文献增加人文素养, 以课 堂提问、师生互动、小组讨论、个人发言等方式, 积 极引导适当鼓励, 潜移默化地帮助学生树立正确的思 想和情感、态度及行为, 形成健康的心理和性格特点。

通过严密的公式、定理推导, 培养学生求真务实、 刻苦钻研的学术态度, 建立学术诚信、实事求是的科 学态度, 甘于寂寞, 甘做冷板発的勇气和定力。通过 创设和知识紧密结合的实际问题并予以解决, 培养学 生的批判性思维，树立创新意识。

\section{2. 具体教学案例解析}

我们以讲授 “线性方程组的应用” 为例, 探索教 学实践中如何采取合理教学方式融入思政元素:

\section{课程教学目标:}

1. 理解并掌握线性方程组解的判断与结构;

2. 了解课程知识在经济学、化学以及网络流等生 产生活中的典型应用。

\section{思政育人目标}

1.坚定学生拥护国家科学发展战略, 满怀爱国 热情, 增强国际视野;

2.树立学生履行时代赋予使命的责任担当, 激 起学生科技强国的理想情怀, 满怀创新精神、钻 研精神和奉献精神;

3. 培养严谨求实的科学精神, 培养刻苦钻研、独 立解决问题的能力和从 0 到 1 的创新意识, 树立正确 的学术观。

\section{教学设计及实施过程}

课堂第一阶段: 介绍性实例

华西里·列昂惕夫, 1973 年诺贝尔经济学奖获得者, 他是投入产出分析方法的创始人。列昂惕夫发展了投 入产出分析方法, 该方法在经济领域产生了重大的作 用。在哈佛大学任教期间, 他写出了一个线性方程组 来描述包含 500 部门的国民经济体系的产出如何分 配给其他经济部门, 因为当时的计算机尚不能处理包 含 500 个方程和未知量的方程组, 列昂惕夫只好把问 题简化为包含 42 个未知量和 42 个方程的方程组。自 此之后, 许多领域的科学家开始利用计算机来分析他 们所建立的数学模型, 由于这些模型中涉及的数据数 量一般都很庞大，模型通常是线性的，也就是说它们 都是用线性方程组来描述的。

现在的科学家和工程师们正在研究大量极其复 杂的科学问题, 随着计算机功能的增强, 线性代数在 应用中的重要性也就迅速增加, 计算机科学在并行处 理和大规模计算方面的快速增长, 使得计算机科学与 线性代数的联系越来越密切。

我们可以再举几个应用的例子:

探测石油: 利用探测船搜寻海底石油时, 计算机 需要每天解上千个线性方程组。

电路: 工程设计师们在利用仿真软件设计电路以 及微芯片时, 它们往往包含数百万个晶体管。这样的 软件技术就需要依赖于线性代数的方法并需要求解 线性方程组。

线性规划: 线性规划模型可以为许多重要的管理 决策提供支持, 这些模型一般都会包含几百个甚至还 要多的变量。

这一阶段教师通过讲解激发学生拥护国家科学 发展战略, 满怀爱国热情, 帮助树立学生履行时代 赋予使命的责任担当, 激起学生科技强国学以致 用的理想情怀, 培养钻研精神和创新精神

课堂第二阶段: 讲解线性方程组解的结构以及具 体实际应用

首先复习知识要点: 线性方程组包括齐次线性方 程组 $A X=0$ 和非齐次线性方程组 $A X=b$, 其中 $A$ 是 系数矩阵, 方程组的解有下列三种情况: 无解、有唯 一解、有无穷多解。 
$\mathbf{a}$ 齐次线性方程组解的结构

设矩阵 $A$ 的秩 $\mathrm{r}(A)=\mathrm{r}$ 。

当 $r=n$ 时, 其中 $n$ 为未知量的个数, $A X=0$ 只有零解;

当 $r<n$ 时, $A X=0$ 有非零解。

设 $x_{1}, x_{2}, \ldots, x_{n-r}$ 是齐次线性方程组 $A X=0$ 的 基础解系, 则 $A X=0$ 的通解为

$k_{1} x_{1}+k_{2} x_{2}+\cdots+k_{n-r} x_{n-r}$,

其中 $k_{1}, k_{2}, \ldots, k_{n-r}$ 为数域 $F$ 上的任意数。

b 非齐次线性方程组解的结构 广矩阵。

设矩阵 $A$ 的秩 $\mathrm{r}(A)=\mathrm{r}(A, b)=\mathrm{r},(A, b)$ 为增

当 $r=n$ 时, $A X=b$ 有唯一解;

当 $r<n$ 时, $A X=b$ 有通解

$x_{0}+k_{1} x_{1}+k_{2} x_{2}+\cdots+k_{n-r} x_{n-r}$,

其中 $x_{0}$ 为方程组 $A X=b$ 的一个特解, $x_{1}, x_{2}, \ldots, x_{n-r}$ 是它的导出组 $A X=0$ 的基础解系, $k_{1}, k_{2}, \ldots, k_{n-r}$ 为数域 $F$ 上的任意数。

课堂本阶段意在通过实例阐述线性方程组有多 个解的情况。

实例 1: 假设某一个经济体系由交通运输、煤炭 制造和林业三个部门组成, 煤炭制造部门销售40\%的 产出给交通运输部门和 $50 \%$ 的产出给林业部门, 余 下产出保留。交通运输部门销售 $60 \%$ 的产出给煤炭 制造部门和 $40 \%$ 的产出给林业部门, 不保留产出。林 业部门销售 $20 \%$ 的产出给煤炭制造部门和 $60 \%$ 的 产出给交通运输业部门, 余下产出保留。

a.试构建该经济体系的产出收支表

b. 试建立线性方程组来表示各部门收支平衡的 条件, 并写出对应的增广矩阵以及解出该方程组。

根据题目描述, 我们可以用符号分别表示煤炭制 造、交通运输以及林业部分的总产出: $P_{\mathrm{m}}, P_{j}, P_{l}$ 并 使得各部门的总产出等于总收入

$$
\left\{\begin{array}{l}
0.4 P_{m}+0.6 P_{l}=P_{\mathrm{j}} \\
0.6 P_{j}+0.1 P_{\mathrm{m}}+0.2 P_{l}=P_{m} \\
0.4 P_{j}+0.5 P_{\mathrm{m}}+0.2 P_{l}=P_{l}
\end{array}\right.
$$
阵

整理该方程组可得到齐次线性方程组的系数矩

$$
\left[\begin{array}{ccc}
-1 & 0.4 & 0.6 \\
0.6 & -0.9 & 0.2 \\
0.4 & 0.5 & -0.8
\end{array}\right]
$$

化系数据矩阵为最简型

$$
\left[\begin{array}{ccc}
1 & 0 & -0.95 \\
0 & 1 & -0.85 \\
0 & 0 & 0
\end{array}\right]
$$

得到通解

$$
\left\{\begin{array}{l}
P_{j}=0.95 P_{l} \\
P_{m}=0.85 P_{l}
\end{array}, P_{l}\right. \text { 为自由未知量 }
$$

实例 2: 化学方程式是用化学式表示化学反应的 式子, 它描述了化学反应的物质消耗以及生产的数量。 比如硫化硼遇水会发生剧烈反应, 生成硼酸和具有臭 鸡蛋味道的硫化氢气体, 化学方程式如下所示:

$$
\mathrm{B}_{2} \mathrm{~S}_{3}+\mathrm{H}_{2} \mathrm{O} \rightarrow \mathrm{H}_{3} \mathrm{BO}_{3}+\mathrm{H}_{2} \mathrm{~S}
$$

为了配平这个方程式, 使得方程式左边的原子总 数等于右边相应的原子总数, 这是因为在化学反应中 每种原子既不会被破坏，也不会被创造。

配平化学方程式的一个系统方法是建立描述化 学反应中每种类型原子数目的向量方程, 由于上述方 程式包含了四种类型的原子, 即嗍 $B$ 、氢 $\mathrm{H}$ 、氧 $\mathrm{O}$, 硫 $\mathrm{S}$, 因此为上述方程式的每一种反应物和生成物构造 一个属于 $R^{4}$ 的向量, 列出如下:

$$
B_{2} S_{3}\left[\begin{array}{l}
2 \\
0 \\
0 \\
3
\end{array}\right], H_{2} O\left[\begin{array}{l}
0 \\
2 \\
1 \\
0
\end{array}\right], H_{3} B O_{3}\left[\begin{array}{l}
1 \\
3 \\
3 \\
0
\end{array}\right], H_{2} S\left[\begin{array}{l}
0 \\
2 \\
0 \\
1
\end{array}\right]
$$

要配平方程式必须满足

$$
x_{1}\left[\begin{array}{l}
2 \\
0 \\
0 \\
3
\end{array}\right]+x_{2}\left[\begin{array}{l}
0 \\
2 \\
1 \\
0
\end{array}\right]=x_{3}\left[\begin{array}{l}
1 \\
3 \\
3 \\
0
\end{array}\right]+x_{4}\left[\begin{array}{l}
0 \\
2 \\
0 \\
1
\end{array}\right]
$$

化简并整理该方程组得到通解

$$
\left\{\begin{array}{l}
x_{1}=\frac{1}{3} x_{4} \\
x_{2}=2 x_{4}, x_{4} \text { 为自由未知量 } \\
x_{3}=\frac{2}{3} x_{4}
\end{array}\right.
$$

一般情况下, 我们会使用尽可能小的正整数来配 平方程式, 所以我们可以取自由未知量 $x_{4}=3$, 于是得 到配平的方程式

$$
\mathrm{B}_{2} \mathrm{~S}_{3}+6 \mathrm{H}_{2} \mathrm{O} \rightarrow 2 \mathrm{H}_{3} \mathrm{BO}_{3}+3 \mathrm{H}_{2} \mathrm{~S}
$$

实例 3: 线性方程组可以用来研究一些数量在网 络中的流动。比如, 当市区道路呈现网格状, 城市规 划及交通工程人员监控其交通流量时, 电气工程师计 算流经电路的电流时, 许多网络中的方程组可能会涉 及到成百乃至上千的变量和方程。

一个网络包含一组称为节点的点集, 以及连接部 分或全部的节点的分支或弧, 流的方向会在每个分支 上有标识, 流量也会有显示或用变量标注。

如下图是某市区一些单行道在某个时间段的交 通流量 (我们以每小时经过的车辆数目计算), 试计 算该网络的车流量。 


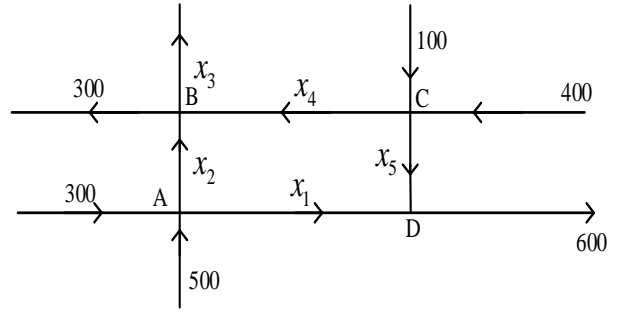

在上图所示的交叉口, 令驶入车辆的数目等于车 辆驶出数目且网络的总流入量等于总流出量, 于是就 能根据上述网络图得到线性方程组并化简得到其增 广矩阵, 化增广矩阵为最简型, 解得网络的车流量为

$$
\left\{\begin{array}{l}
x_{1}=600-x_{5} \\
x_{2}=200+x_{5}, x_{5} \text { 为自由未知量, } \\
x_{3}=400, \\
x_{4}=500-x_{5}
\end{array}\right.
$$

网络分支中的一个负值流量对应于模型中显示 方向相反的流量, 由于在本问题中的道路是单行线, 故而不允许负值存在, 此情况给变量的可能取值会增 加某种限制。

限于篇幅, 以上所列实例的详细解答过程在本文 中有省略。

这一阶段教师主要通过讲解和知识紧密结合的 实际问题, 积极引导小组讨论、集体或个人发言, 鼓 励学生自主探究、交流, 通过探究体验、论证建立方 程组的过程, 培养严谨求实的科学精神, 增强学生数 学思维的实际应用能力, 解决生产生活中的问题, 教 师通过正面引导, 增强学生面对未知问题的胆识和勇 气 (思想与感情、态度与行为、心理与性格)。以求增 强学生的探索欲望和成就感, 增强自信心, 培养自主 思考能力和创新能力。

\section{课堂第三阶段：巩固练习}

本阶段旨在通过练习巩固所学, 提高学生学习积 极性, 培养学生认真刻苦、独立探究解决问题的能力 和从 0 到 1 的创新意识, 并在求真务实中逐步树立正 确的学术观、人生观、价值观。

练习 1 设 $x_{1}, x_{2}$ 是非齐次线性方程组 $A X=b$ 的两个特解, 又 $y_{1}, y_{2}$ 是对应导出组 $A X=0$ 的一 个基础解系, $k_{1}, k_{2}$ 为任意常数, 则非齐次线性方程 组 $A X=b$ 的全部解为:

$$
\begin{gathered}
A . \frac{x_{1}-x_{2}}{2}+k_{1} y_{2}+k_{2}\left(y_{1}-y_{2}\right), \\
B . \frac{x_{1}+x_{2}}{2}+k_{1} y_{2}+k_{2}\left(x_{1}-x_{2}\right), \\
C . \frac{x_{1}+x_{2}}{2}+k_{1} y_{2}+k_{2}\left(y_{1}+y_{2}\right), \\
D . \frac{x_{1}-x_{2}}{2}+k_{1} y_{2}+k_{2}\left(x_{1}-x_{2}\right) .
\end{gathered}
$$

练习 2 设有 $n+1$ 个人以及供他们读的 $n$ 种小 册子, 假定每一个人已经读了其中一些小册子 (至少 读了一本), 试说明这 $n+1$ 个人中必可以找出这样
两组人使得其中一组人读过的小册子与另外一组人 读过的小册子种类相同。即一组人每人读过的小册子 合在一起, 其种类与另外一组人每人读过的小册子合 在一起的种类相同。

\section{3. 组织线上（下）阅读书会，增加课程温 度}

数学作为一门基础学科, 对很多人来说是枯燥的、 深奥的、抽象的, 但其数学知识背后的人物 (数学家) 却是有血有肉、有温度、有故事、有情怀的, 为了让 学生更好地了解数学, 走近数学更走进数学, 我们通 过建立线上阅读讨论群或利用课外时间固定一个地 点安排读书会的形式, 定时推送相关数学家的故事、 有关数学的书籍, 比如 《数学与生活》 (远山启著, 吕 砚山等译, 人民邮电出版社)、《玩不够的数学》(让保罗.德拉耶著, 路遥译, 人民邮电出版社) 等, 以及 推送与课程相关的前沿文章, 用具有价值引领的人物 和故事, 提高学生对专业学习的积极性, 使学生能够 充分投入到专业学习中, 树立正确人生观价值观世界 观, 通过阅读文献和书籍这本身就是思政融入课堂的 一种手段, 同时也能提升学生的人文素养, 增强师生 之间、生生之间的互动性, 通过讨论甚至辩论激发热 情交流思想, 鼓励学生畅所欲言撰写心得 ${ }^{[4-5]}$ 。

\section{3. 结论}

蔡元培先生说过 “教育是帮助被教育的人, 给他 们能发展自己的能力, 完成他的人格, 于人类文化上 能尽一份子责任”, 专业课课程思政在设计上要充分 考虑价值高度、专业深度以及两者的关联度, 但 “课 程思政 $\neq$ 专业课+鸡汤”, 思政融入课堂需要老师长期 的坚守和日常工作生活中的言传身教, 它不是一件立 竿见影的事情, 这就像春天播下的一粒种子, 不能指 望它一夜就能发芽开花, 但是, 每一节课上的积极引 领, 每一次课后的阅读交流, 每一回的思想碰撞, 这 些点滴留给学生的印记和影响一定会草蛇灰线, 伏脉 于千里之外。

教育 “就像一棵树摇动另一棵树, 一朵云触碰另 一朵云, 一个灵魂唤醒另一个灵魂” 我们要深信: 一 颗星星是渺小的, 但一群星星是闪亮的。

\section{项目基金}

本文为国家自然科学基金(11701252), 临沂大学 博士科研启动基金(LYDX2016BS080)的阶段性成果 之一。

\section{REFERENCES}

[1] Liu Y.,(2021) Optimization design of Linear Algebra course content based on ability training,Heilongjiang Science,3:78-79. 
[2] Zhang L.,Wei L.,(2020) Exploration of “ Linear Algebra" teaching of computer specialty in the background of engineering education certification, The Guide of Science \& Education, 8:118-119.

[3] David C.Lay, Steven R.Lay, Judi J.McDonald, Linear Algebra and Its Applications Fifth Edition, China Machine Press.

[4] Hu Y.,(2020) Student centeredonline-teaching for Linear Algebra course,3:75-79, China Education of Light Industry.

[5] Deng R., Chen Q., Li Y., (2020) Implementation of Ideological and Political Education in College Mathematics, 3:100-104,Journal of Ningbo University of Technology. 